\title{
Augmented data
}

Juan Jiménez Recaredo

$10 / 4 / 2021$

\section{Resumen:}

Se trata de dos muestras independientes: una con voluntarios que utilizaron la mascarilla sin el dispositivo (Controles) y otra con voluntarios que realizaron la prueba con el dispositivo diseñado en ADEMA (Intervención).

Los grupos son homogéneos en la distribución de hombres y

mujeres, sin que haya podido encontrar diferencias estadísticamente significativas en las medianas de las edades ni en las medianas de la sensibilidad al olor, medida según el número de activaciones en el test inicial.

La proporción de voluntarios del grupo intervención que no detecta ningún olor es significativamente mayor que la del grupo control $(\cap(p-$ valor $=9.3 \backslash$ cdot $\left.10^{\wedge}\{-25\} \backslash\right)$ ), para una potencia del contraste $=100 \%$

\section{Comparación de los grupos:}

Como paso inicial hay que asegurar que los grupos (después de eliminar del análisis los voluntarios que no fueron evaluados en la prueba) son comparables en cuanto a las variables características de cada uno: 1) las distribuciones de hombres y mujeres, 2) las de las edades y 3) la sensibilidad inicial medida por el número de activaciones.

\section{Comparación por Género}

La comparación de las distribuciones del Género según el grupo se 
puede hacer, o bien comparando las proporciones apropiadas (que fue lo que presenté en el análisis anterior), ó bien se pueden hacer los test de homogeneidad \(|chi^2〉).

En el primer caso, tomando en cuenta que luego de las modificaciones hechas el grupo Control está compuesto por 28 hombres y 33 mujeres, mientras que en el grupo Intervención hay 25 hombres y 31 mujeres, los resultados de las comparaciones de proporciones usando el test exacto de Fisher (este es el test que debe ser usado porque las muestras son pequeñas) son:

\begin{tabular}{|c|c|c|c|c|}
\hline Variable & $\begin{array}{l}\text { Contr } \\
\text { ol }\end{array}$ & $\begin{array}{l}\text { Interventi } \\
\text { on }\end{array}$ & $\begin{array}{c}\mathrm{p}- \\
\text { valor }\end{array}$ & $\begin{array}{c}\mathrm{Cl}(95 \%) \text { (for the Odds } \\
\text { ratio) }\end{array}$ \\
\hline $\begin{array}{c}\text { Males (totals and } \\
\%)\end{array}$ & $\begin{array}{l}28, \\
46 \%\end{array}$ & $25,45 \%$ & 1 & (0.48 2.3) \\
\hline $\begin{array}{c}\text { Females (totals } \\
\text { and } \% \text { ) }\end{array}$ & $\begin{array}{l}33, \\
54 \%\end{array}$ & $31,55 \%$ & 1 & (0.43 2.1) \\
\hline
\end{tabular}

De manera que no hay diferencias significativas entre la proporción de hombres en el grupo control y la proporción en el grupo intervención. Lo mismo vale para la comparación de proporciones de mujeres entre ambos grupos.

Los detalles son:

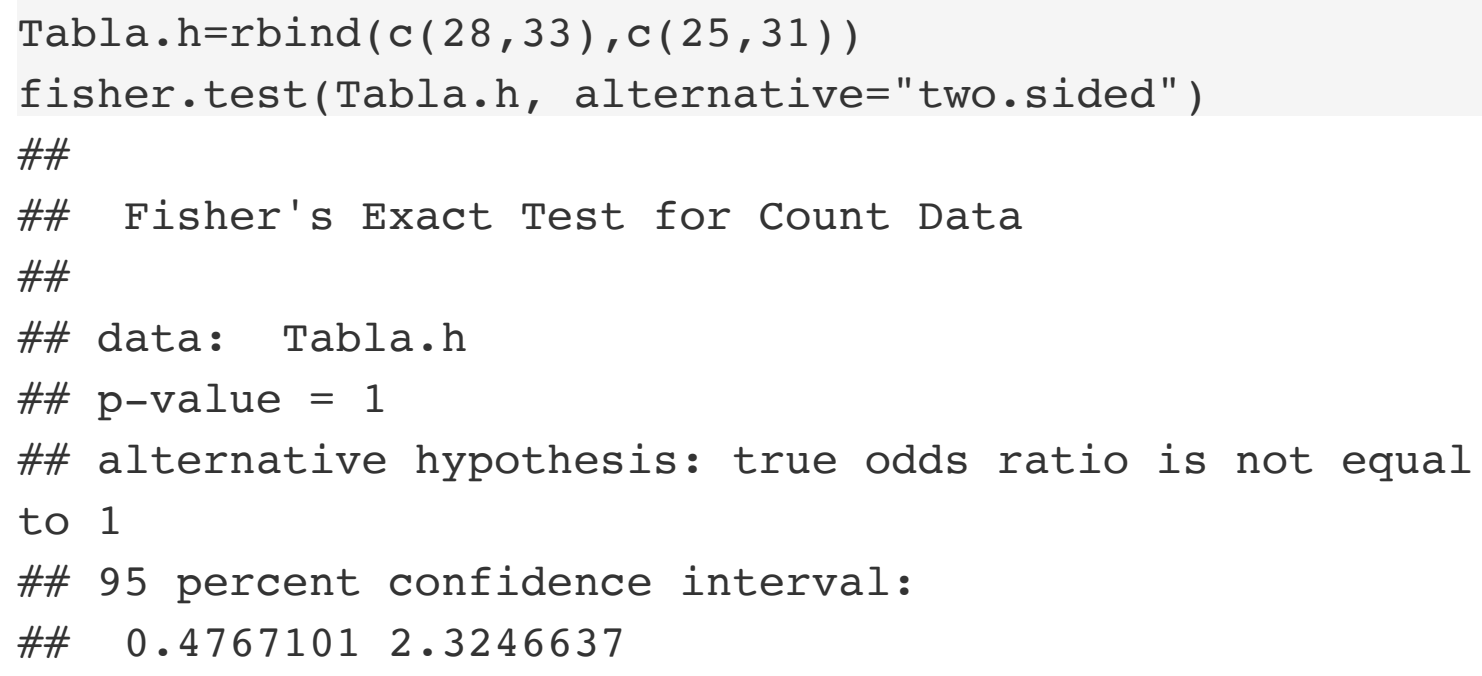




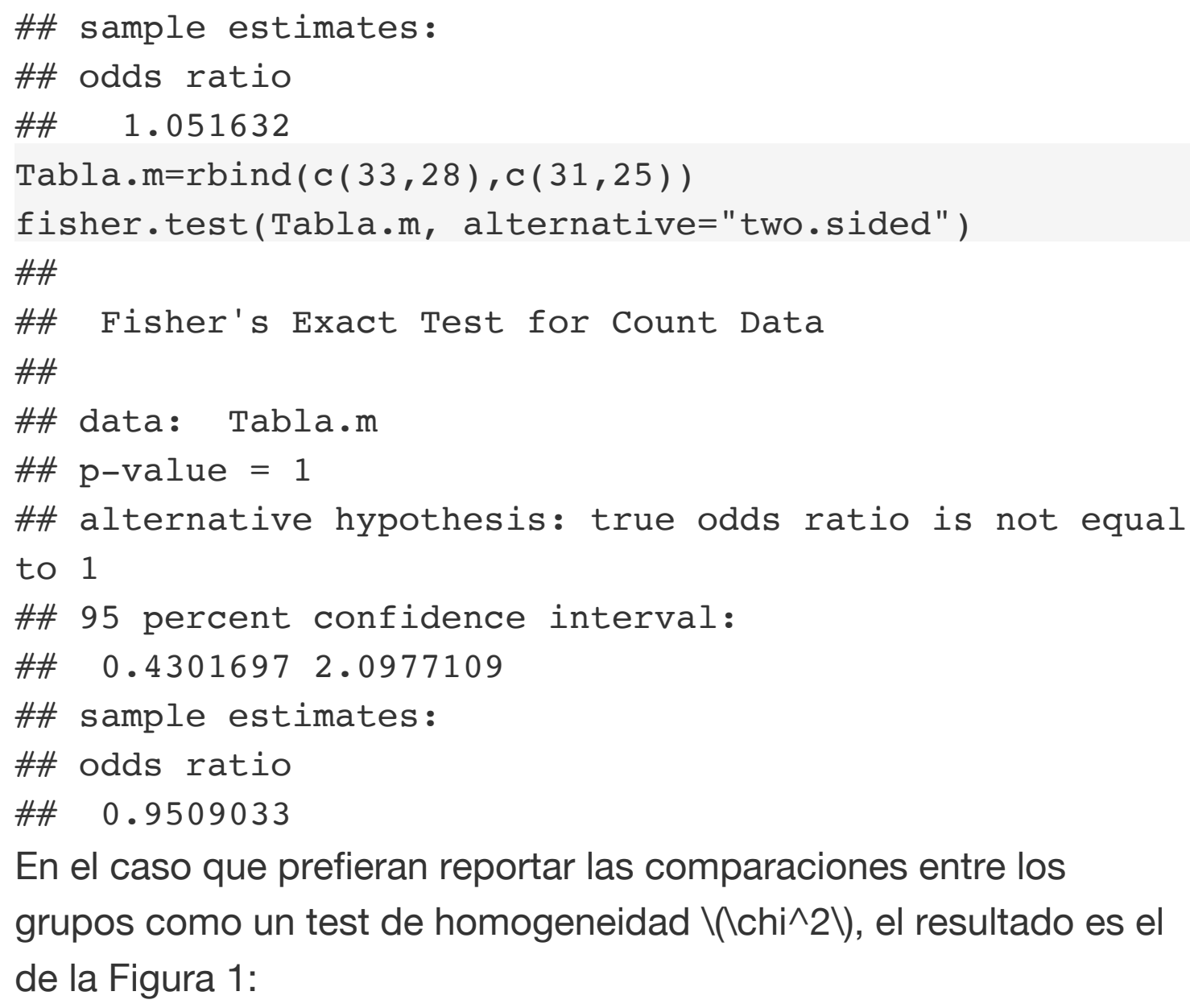
de la Figura 1: 
$\chi_{\text {Pearson }}^{2}(1)=0.02, p=0.891, \widehat{V}_{\text {Cramer }}=0.00, \mathrm{Cl}_{95 \%}[-0.12,0.07], n_{\text {obs }}=117$
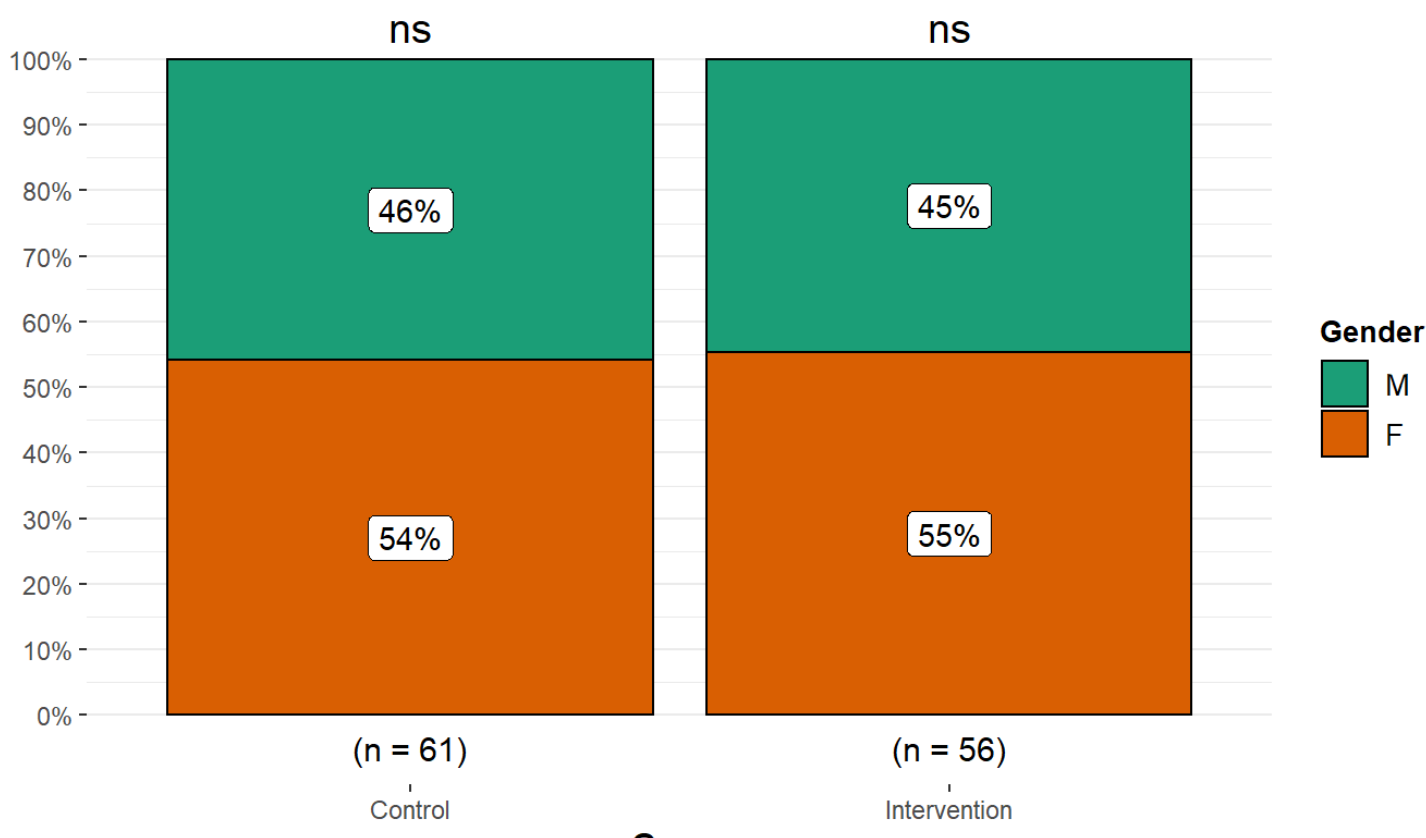

Group

$\log _{e}\left(\mathrm{BF}_{01}\right)=1.47, a=1.00$

Figura 1: Distribución de la variable Género en los grupos:

Realizamos un test \(|chi^2\) para analizar la homogeneidad en la distribución del Género entre los dos grupos y, como pueden ver en la leyenda, tanto la estimación puntual del estadístico como el intervalo de confianza, indican que no se puede rechazar la hipótesis nula de que el grupo Control y el grupo Intervención son comparables en lo que se refiere a la distribución del Género ( $p$-valor $=0.891)$.

\section{Comparación por edades:}

En cuanto a la comparación de las edades entre los dos grupos, los resultados son los que se indican en la Figura 2. Para la evaluación de la normalidad en la distribución de las edades en ambos grupos, el test usado es el de Kolmogorov-Smirnov (con la corrección 
Lilliefors) que está implementado en la librería nortest de $\mathrm{R}$ y que se aplica mediante la función lillie.test(). Para cualquier referencia adicional ver: https://cran.r-project.org/web/packages/nortest/ nortest.pdf. Los resultados del test de normalidad para la distribución de edades en los grupos control e intervención son:

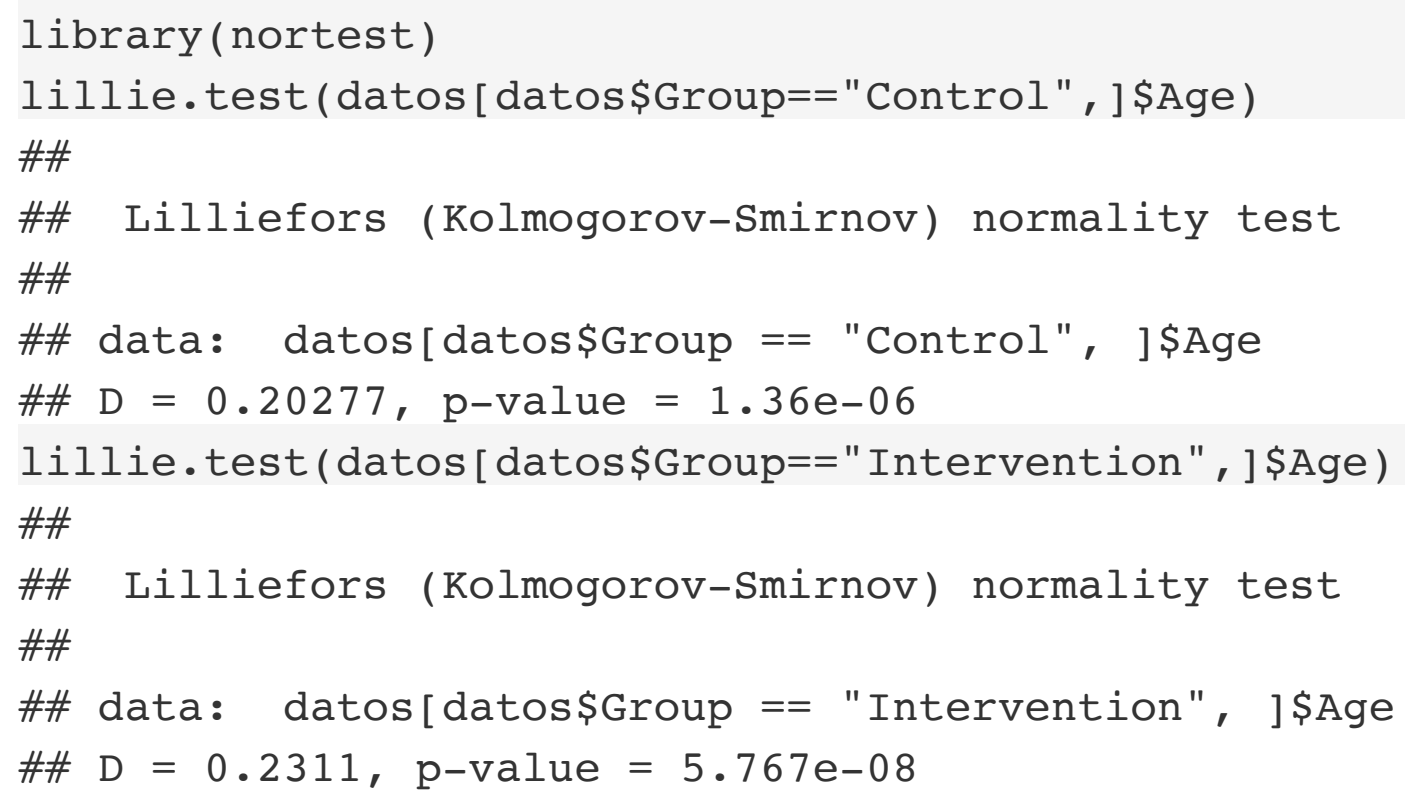

Como pueden ver, ambos $p$-valores son menores a 0.05 , por lo que la hipótesis nula (los datos se distribuyen normalmente) debe ser rechazada. 


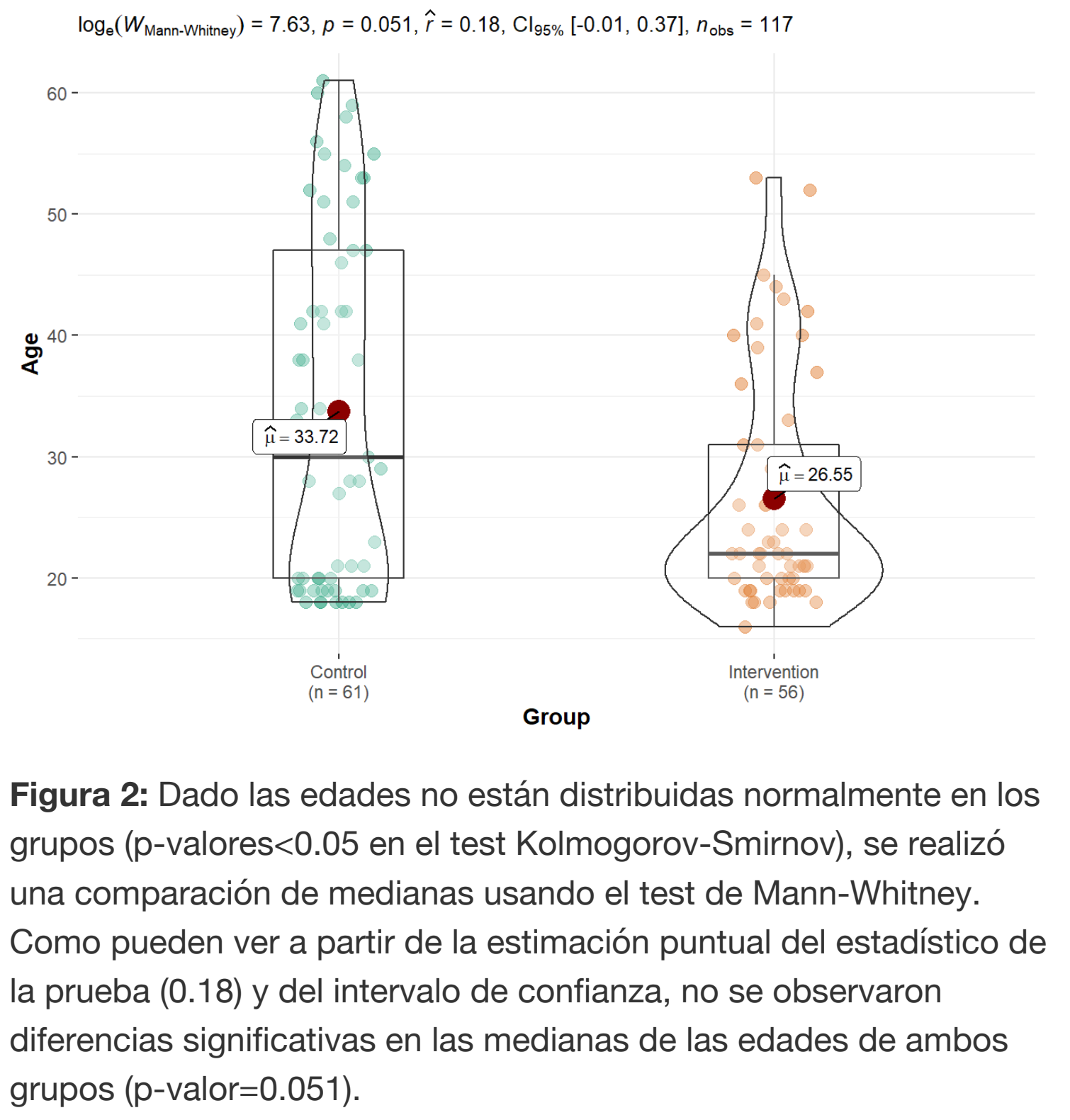

\section{Comparación de la sensibilidad al olor en el test inicial.}

Lo primero es hacer el test de normalidad para la sensibilidad medida antes de la prueba:

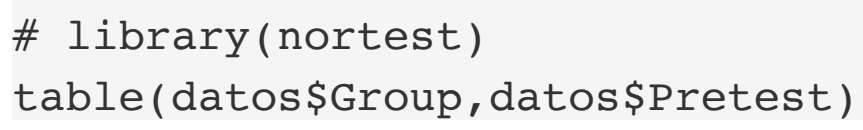




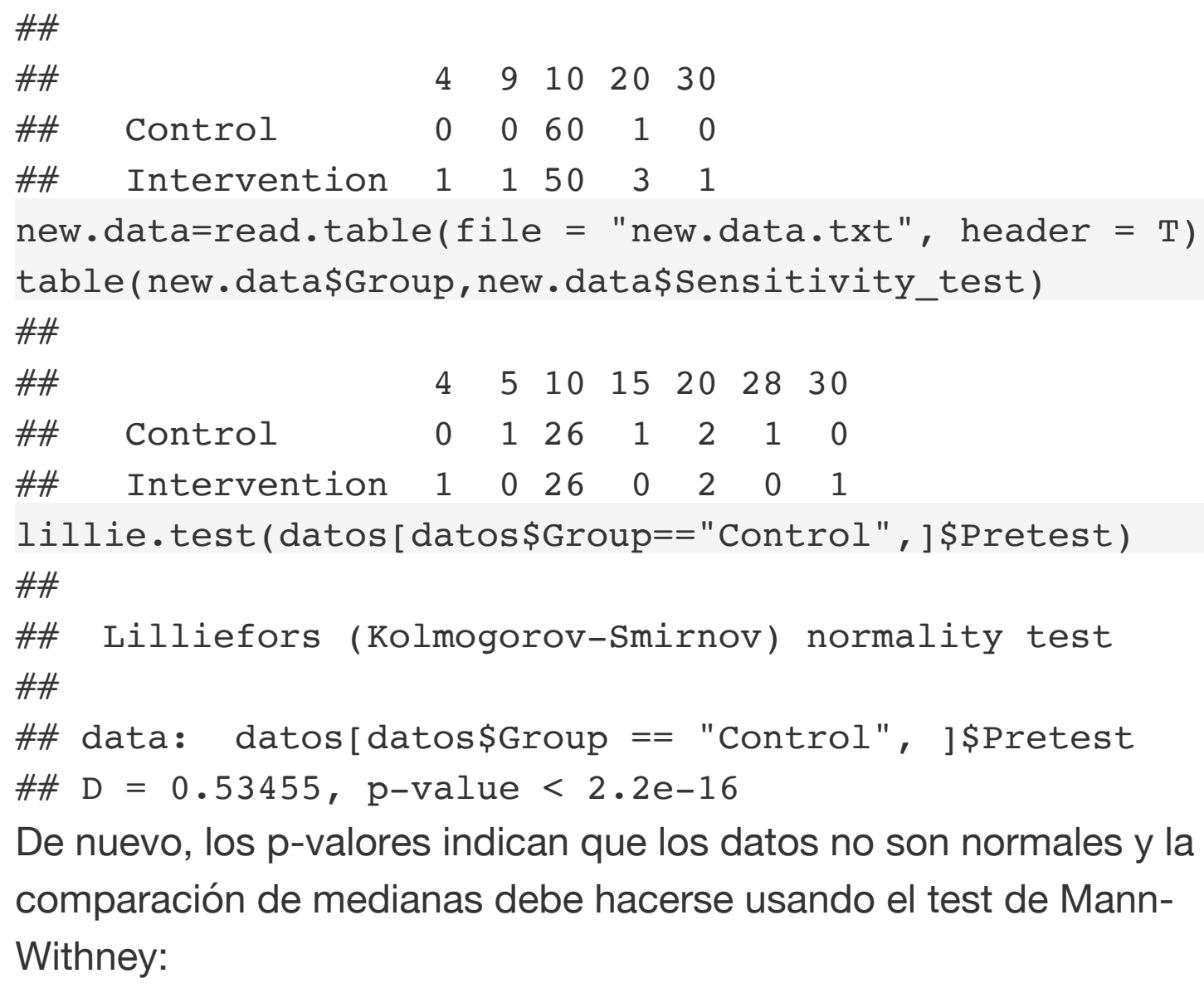




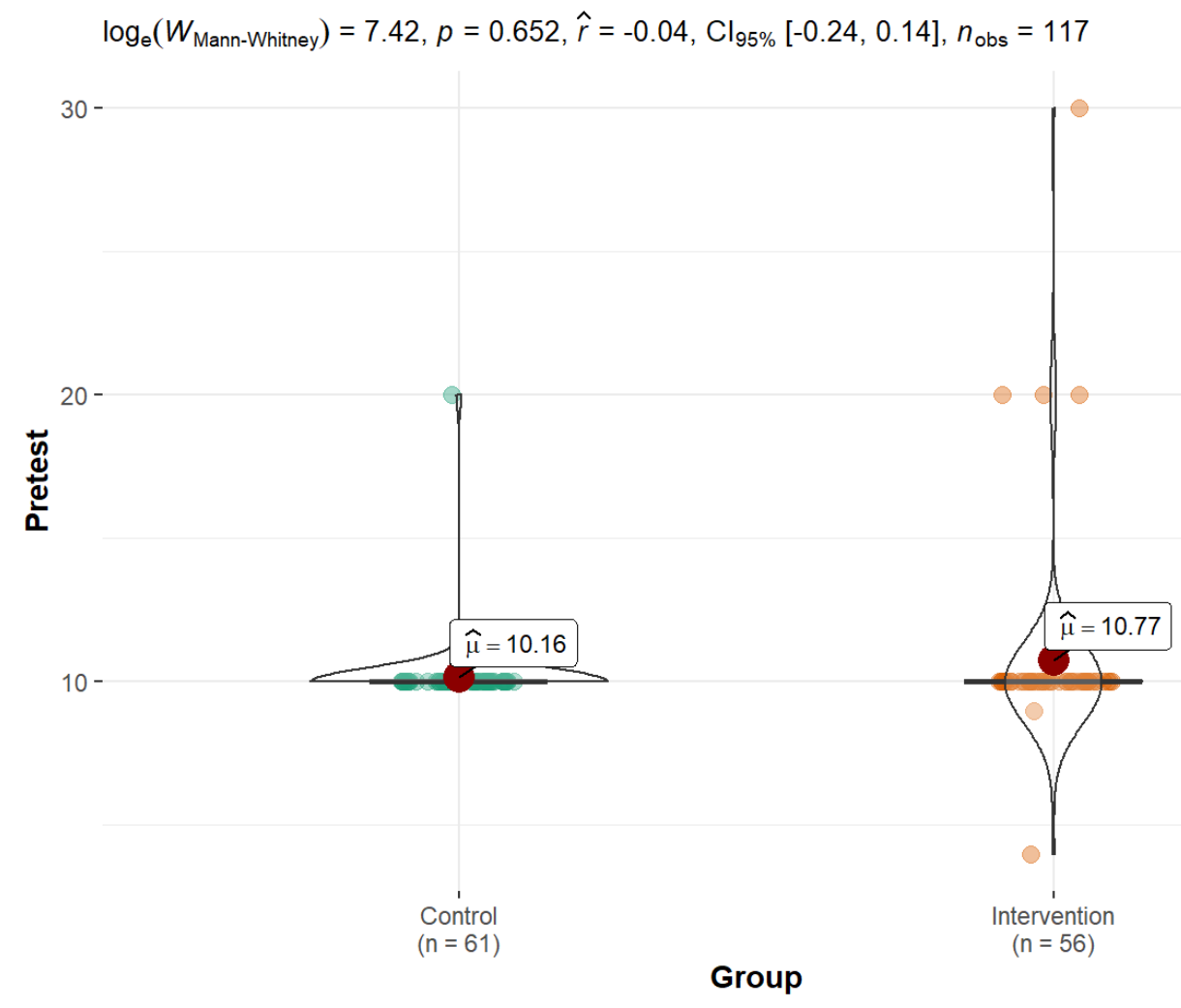

Figura 3: Los números de activaciones en la prueba inicial de sensibilidad no están distribuidos normalmente en los grupos ( $p$ valores $<0.05$ en el test Kolmogorov-Smirnov), por lo que se realizó una comparación de medianas usando el test de Mann-Whitney. Al igual que en el caso de las edades, no se observaron diferencias significativas en las medianas del número de activaciones de ambos grupos ( $p$-valor=0.652).

\section{Análisis de la prueba}

Una vez aclarado que los dos grupos son comparables, hay que entonces que comparar la distribución de APPROVED (los que no sintieron olor) con la de los FAILED (los que sintieron olor). De nuevo, esto se puede hacer comparando las proporciones entre los grupos control e intervención (usando el test exacto de Fisher), o con un test 
((chi^2).

En el primer caso hay que tomar en cuenta que:

table (datos\$Group, datos \$Outcome)

\#\#

\#\# Approved Failed

\#\# Control $\quad 0 \quad 61$

\#\# Intervention $53 \quad 3$

prop.table (table (datos\$Group, datos\$Outcome), margin =

1)

\#\#

\#\# Approved Failed

\#\# Control 0.000000001 .00000000

\#\# Intervention $0.94642857 \quad 0.05357143$

es decir, que de los $\backslash\left(n_{-} \mathrm{i}=56\right)$ voluntarios que hicieron la prueba con el dispositivo, hubo 53 que no sintieron el olor (un 95\%), mientras que de los $\backslash\left(n \_c=61 \backslash\right)$ voluntarios que hicieron la prueba sin el dispositivo, todos lo sintieron.

En este caso, el test de comparación de proporciones (test exacto de Fisher) da como resultado $\backslash\left(p-v a l o r<2.2 \backslash\right.$ cdot $10^{\wedge}\{-16\}, C I \_\{95 \backslash \%\}$ $=(203$, linfty $) \backslash$ ) (recuerden que en el test de Fisher no se puede calcular el Cl para la proporción sino para el Odds ratio)

Detalles:

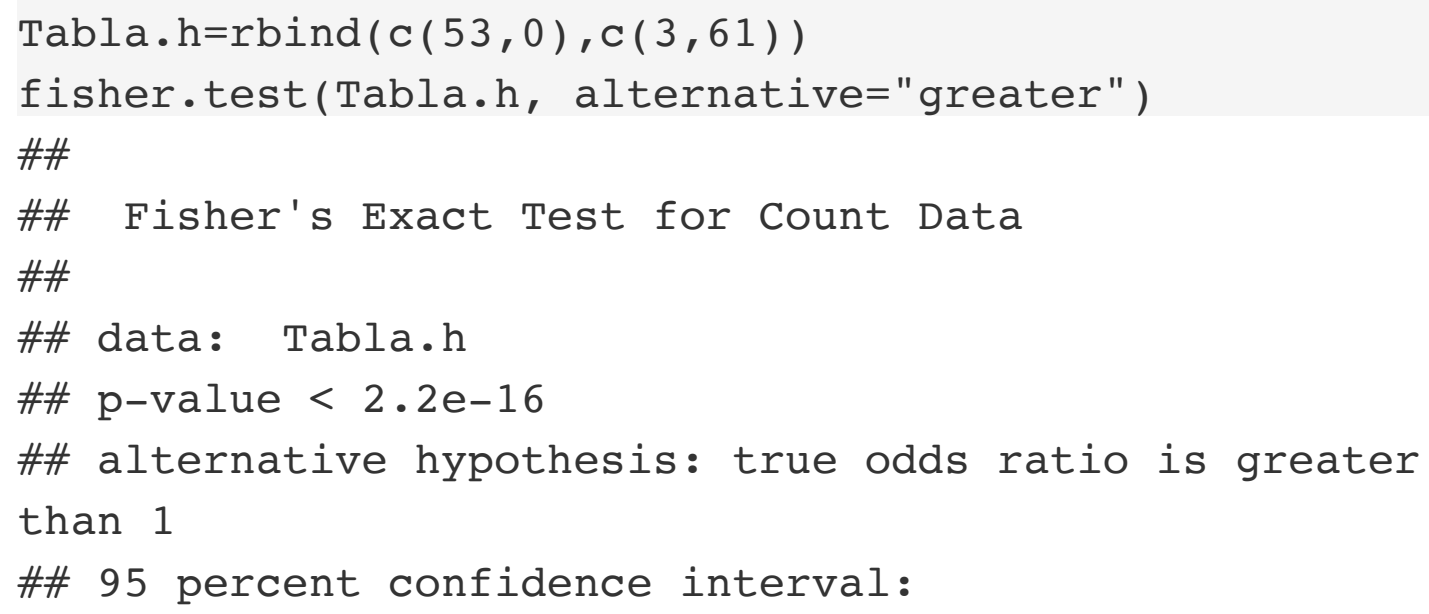




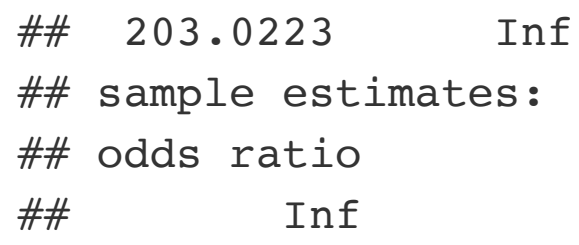

Finalmente, si prefieren hacer el análisis con un test de homogeneidad:

$$
\chi_{\text {Pearson }}^{2}(1)=105.54, p=9.29 \mathrm{e}-25, \widehat{V}_{\text {Cramer }}=0.95, \mathrm{Cl}_{95 \%}[0.90,1.01], n_{\text {obs }}=117
$$

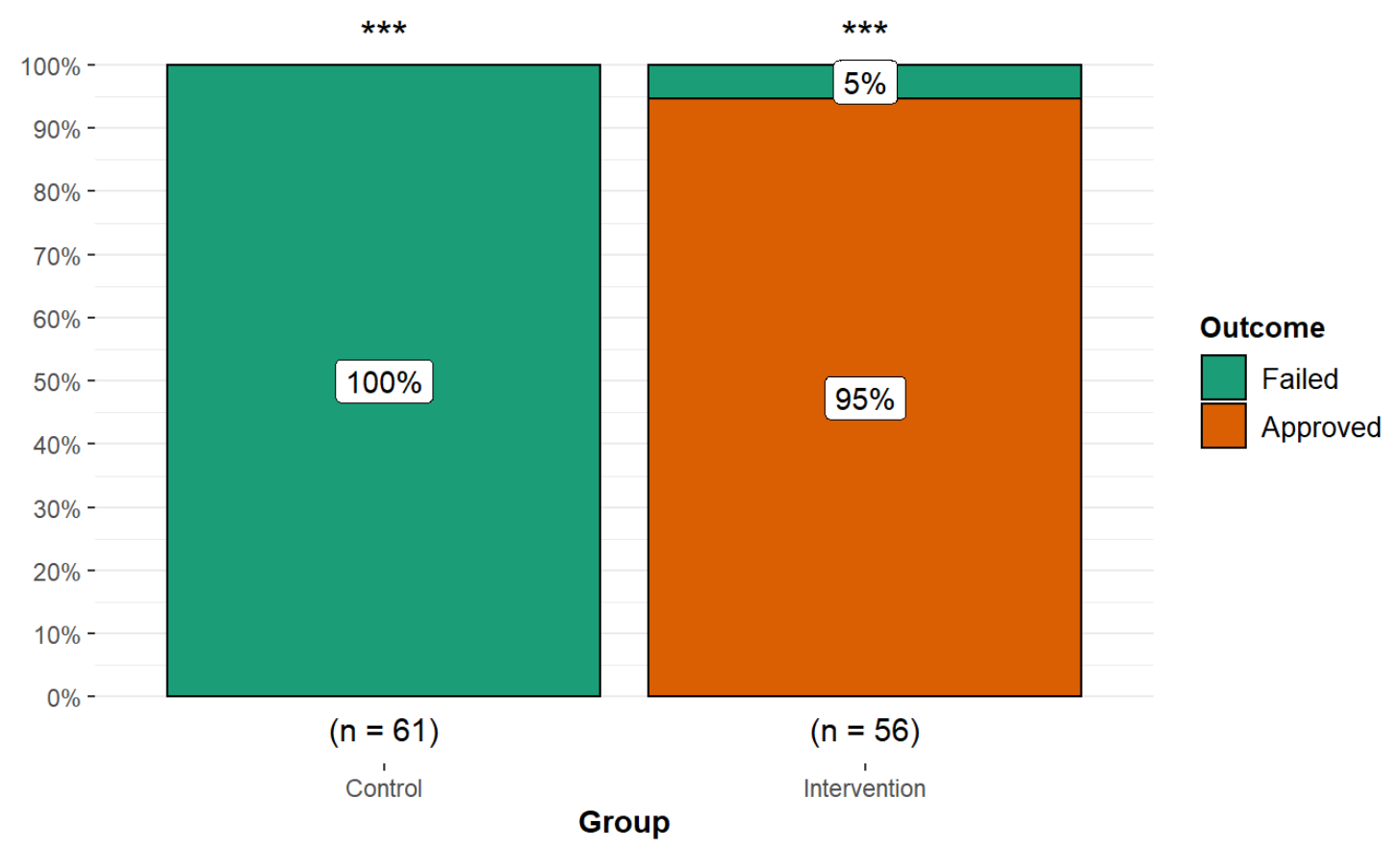

$\log _{\mathrm{e}}\left(\mathrm{BF}_{01}\right)=-64.35, \mathrm{a}=1.00$

Figura 4: Distribución del resultado de la prueba en los grupos: Realizamos un test $\backslash\left(\mathrm{chi}^{\wedge}{ }^{\wedge} 2\right)$ para analizar la homogeneidad en la distribución de la respuesta entre los dos grupos y resulta un coeficiente de Cramer $\left.=0.95 \cap\left(C I \_\{95 \backslash \%\}=(0.85,1.02) \backslash\right)\right)$, con lo que se debe rechazar la hipótesis nula de que no hay diferencias en la distribución del resultado de la prueba entre el grupo Control y el 
grupo Intervención $(p$-valor $=\backslash(9.3 \backslash$ cdot $10 \wedge\{-25\}\rangle))$.

La potencia del contraste, para un nivel de significancia 0.05 , vuelve a ser del 100\%:

library (pwr)

ES.h $(0.95,0)$

\#\# [1] 2.690566

pwr. $2 \mathrm{p} 2 \mathrm{n}$.test $(\mathrm{h}=2.606, \mathrm{n} 1=61, \mathrm{n} 2=56$,

sig. level=0.05, power=NULL, alternative="greater" ) \$power \#\# [1] 1 